\title{
Macroinvertebrados y fitoplancton como bioindicadores de contaminación en la cuenca del río Chicama, Perú
}

\section{Macroinvertebrates and phytoplanckton as bioindicators of pollution in Chicama river basin, Peru}

\author{
Santos R. Santillán-Aredo ${ }^{1}$, Ana M. Guerrero-Padilla²
}

Fecha de recepción: 24 de enero de 2018 Fecha de aprobación: 6 de mayo de 2018

Santillán-Aredo, S; Guerrero-Padilla, A. Macroinvertebrados y fitoplancton como bioindicadores de contaminación en la cuenca del río Chicama, Perú. Tecnología en Marcha. Vol. 31-4. Octubre-Diciembre 2018. Pág 97-110.

https://doi.org/10.18845/tm.v31i4.3968

1 Biólogo egresado de la Facultad de Ciencias Biológicas, Universidad Nacional de Trujillo-Perú. Correo electrónico: rivert_89@hotmail.com

2 Doctora en Ciencias e Ingeniería de la Facultad de Ciencias Biológicas, Universidad Nacional de Trujillo-Perú. Correo electrónico: mguerrero@unitru.edu.pe 


\title{
Palabras clave
}

Bioindicadores; Trent Biotic Index; Chandler Biotic Score.

\section{Resumen}

El objetivo principal fue evaluar la población de macroinvertebrados y fitoplancton como bioindicadores de contaminación en la cuenca del río Chicama, Perú. Se ubicó cuatro estaciones; para macroinvertebrados se evaluó mediante el Trent Biotic Index y Chandler Biotic Score, estableciendo la relación entre las variables (abundancia de taxones) con el coeficiente de correlación de Spearman. La evaluación del fitoplancton se realizó mediante el índice saprobio propuesto por Pantle y Buck. Asimismo, se determinó el índice de Shannon Weaver $\left(H^{\prime}\right)$ y el índice de Simpson ( $\left.D^{\prime}\right)$. La fauna bentónica con 24 géneros, 18 familias y 9 ordenes, predominancia de Baetis sp., Rhagovelia sp. y Atopsyche sp. El fitoplancton estuvo constituido principalmente por diatomeas (Bacillariophyta) y algunas especies pertenecientes a la clase Chlorophyta, con predominancia de Nitzschia linearis, Fragilaria capucina y Gomphonema sp. Finalmente de los índices evaluados, Trent Biotic Index y Chandler Biotic Score, se determinó contaminación leve a moderada de acuerdo al índice de Pantle y Buck.

\section{Keywords}

Biondicators; Trent Biotic Index; Chandler Biotic Score.

\begin{abstract}
The principal objective was to assess phytoplankton and macroinvertebrates as bioindicators of pollution in Chicama river basin, Peru. Was developed four stations and was assess macroinvertebrates by Trent Biotic Index and Chandler Biotic Score, and it was assess the abundance of taxa related to the variables measures, to establish the relationship between the variables with the Spearman correlation coefficient. The assessment of the phytoplankton are carried out through the index proposed by Pantle and Buck; similarly, the index was calculated from Shannon Weaver $\left(H^{\prime}\right)$ and the index of Simpson $\left(D^{\prime}\right)$. It was registered 24 genera of benthuc fauna, 18 families and 9 orders, with a predominance of Baetis sp., Rhagovelia sp. and Atopsyche sp. and to a lesser degree, in comparison with its predecessors, Tricorythodes sp., Clinotanypus sp., and Simulium sp. In regards phytoplankton was composed mainly by diatoms (Bacillariophyta) and some species belonging to the class Chlorophyta, with predominance of Nitzschia linearis, Fragilaria capucina and Gomphonema sp. Finally, the indices evaluated, Trent biotic index and Chandler Biotic Score determined that there is from slight to moderate pollution according to the index of Pantle and Buck.
\end{abstract}

\section{Introducción}

Los macroinvertebrados y el fitoplancton son componentes importantes tanto en la biodiversidad como en el funcionamiento de los ecosistemas dulceacuícolas [1][2], mediante las cuales se estudiaron las respuestas de los organismos que habitan el medio acuático para evaluar la calidad de agua, estableciendo el concepto de indicador biológico [3]. A diferencia de los análisis fisicoquímicos que determina condiciones actuales, con el uso de bioindicadores brindan las condiciones pasadas y actuales con mayor precisión [4] [5]. Debido a que en los ecosistemas acuáticos, en condiciones normales, se presentan muchas especies y no solo una, es necesario analizar la estructura de la comunidad para caracterizar al ambiente 
que la rodea, en tal sentido las especies individuales llegan a tener importancia en casos de contaminación intermedios [6] [7]. Se ha desarrollado métodos rápidos de evaluación de agua usando macroinvertebrados como bioindicadores [8]; en España se adoptó el uso de macroinvertebrados acuáticos en los programas de evaluación de calidad de agua [9]; la perturbación en las corrientes de agua se califican en relación con las características de las especies de macroinvertebrados y riqueza de dichas especies [10]. Este tipo de estudios se ha realizado en Norteamérica [11] [8] [12], en Inglaterra [13] [14] y en Europa se está empleando las metodologías dentro de la Directiva Marco del Agua [15]. Especialmente el uso de macroinvertebrados en sus diversos métodos permiten evaluar e identificar de manera rápida y precisa, la relación causal entre los cambios específicos de la calidad de agua y su relación con especies tolerantes, esto debido a sus ventajas como organismos bioindicadores de contaminación, sus ciclos de vida relativamente cortos comparados con los peces, lo que refleja con mayor rapidez las alteraciones del río mediante cambios en la estructura de sus poblaciones y comunidades; el lugar donde habitan y se alimentan, el cual se encuentra en o sobre los sedimentos donde tienden a acumularse las toxinas; su sensibilidad a los factores de perturbación y su respuesta a las sustancias contaminantes presentes tanto en el agua como en sedimentos; así como su amplia distribución, abundancia y fácil recolección debido a su tamaño, el que los hace visibles a simple vista [16] [7] [4].

Por otro lado, el fitoplancton comprende organismos muy antiguos que prácticamente habitan en todos los ecosistemas del planeta, presentando amplia gama de tamaños, formas y estrategias ecológicas y fisiológicas [17]. Dado sus cortos ciclos vitales, que le permiten responder rápidamente a los cambios ambientales, la dependencia sobre las condiciones físicas e hidrológicas del agua, la composición química del agua, y los factores biológicos, los cuales son capaces de reducir densas poblaciones fitoplanctónica, hacen del fitoplancton, organismos adecuados para la detección y seguimiento de las presiones fisicoquímicas relacionadas con: contaminación térmica, cambios en la mineralización del agua, eutrofización, contaminación orgánica (soluble y particulada), así como bioindicadores de las presiones hidromorfológicas que determinan cambios en la tasa de renovación de las masas agua [17]. En tal sentido, desde hace algunas décadas es reconocida la importancia del uso de estas algas como indicadores biológicos, a través de métodos ecológicos (riqueza específica, diversidad, índice saprobio y análisis multivariados), instalándose de esta manera un importante número de programas de monitoreo biológico establecidos a nivel mundial para valorar las condiciones ambientales de ríos, lagos, estuarios y humedales [18].

En Perú, las evaluaciones de calidad de aguas en las cuencas hídricas se basa principalmente en la valoración fisicoquímica, microbiológica y biológica del agua, este último principalmente respecto a los macroinvertebrados registrándose entre los primeros trabajos realizados el uso de los macroinvertebrados en la determinación de la calidad de agua del río Rímac [19] [20]; en el río Moche [21]; y la cuenca del río Chicama [22] [23] [24]. Con respecto a la comunidad fitoplanctónica no se han presentado estudios, que indiquen su utilización como indicadores biológicos de contaminación en cuerpos de agua. Sin embargo, se han realizado estudios en otros países del continente americano, entre los que podemos mencionar en México [25], y en Argentina [26] [27] [28]. Actualmente la calidad ambiental de las cuencas se encuentran en un estado de degradación general, donde las acciones de los seres humanos afectan ostensiblemente a un sinnúmero de ecosistemas acuáticos, perjudicando la integridad de los atributos ecológicos de los sistemas naturales acuáticos y terrestre, y modificando con ello la evolución natural de los mismos en diferentes escalas. El río Chicama, uno de los más importantes ríos del país, determinante por irrigar grandes áreas de cultivos en la parte inferior del sector central de la región La Libertad, en los predios históricos como: Casa Grande, Cartavio, Sintuco, Chiquitoy, Roma, Chiclín; y extensiones de agricultores individuales en Paiján, 
Chocope, y Ascope; el cual viene siendo afectado por numerosas alteraciones de origen antrópico como: vertidos de numerosas pequeñas mineras informales, vertidos orgánicos de poblaciones rurales, regulación de caudales por el uso agrícola, alteración del bosque de la rivera y el movimiento de suelo agrícolas; conllevado aun deterioro paulatino de dicha cuenca [22]. Dada la creciente preocupación por la sustentabilidad del ambiente y del franco deterioro en que se encuentran los ecosistemas, por los fenómenos naturales, la influencia del hombre en los cambios antrópicos que éste genera y la falta de instrumentos de base biológica que sirvan de patrón de referencia para la evaluación en la determinación de la calidad ambiental el uso de bioindicadores en la determinación de la contaminación de la cuenca del río Chicama, Perú.

\section{Metodología}

El área de estudio se ubicó en la cuenca del río Chicama, la cual se emplaza en la zona norte del Perú, y pertenece a la hoya hidrológica del Océano Pacífico, está ubicada en la región La Libertad y de la región de Cajamarca. Posee una longitud aproximada de $115 \mathrm{~km}$, extendiéndose desde el nivel del mar hasta sus nacientes en los andes occidentales, de los cuales se tomaron 47,5 km, cuyas coordenadas geográficas de sus puntos extremos se encuentran comprendidos aproximadamente entre los paralelos 719473 E y 741199 E y los meridianos 9145708 S y 9162047 S respectivamente. La presente investigación se realizó evaluaciones en cuatro estaciones de muestreo (figura 1), determinando parámetros fisicoquímicos mediante el método estandarizados [29]. Se evaluaron los parámetros hidrobiológicos: macroinvertebrados, fitoplancton, la toma de muestra de macroinvertebrados se realizó dos repeticiones por estación de muestreo, mediante el método multihabitad y la técnica rápida [30], utilizando una red "D-net" con malla de $300 \mu \mathrm{m}$ de abertura, la identificación se realizó en la Universidad Nacional de Trujillo según métodos [31] [32], empleando las claves taxonómicas [33] [34] [35] [36]. En la evaluación de los macroinvertebrados se determinó el Trent Biotic Index, Chandler Biotic Score, y la aplicación del índice propuesto por Pantle y Buck [37].

Para el fitoplancton, se aplicó el coeficiente de correlación de Spearman $\left(r_{s}\right)$ y su correspondiente test de significancia, para establecer relación entre el índice y las variables físico-químicas y biológicas objeto de estudio [38]. Se determinó el índice de Shannon Weaver [18], cuya expresión es $\mathrm{H}^{\prime}=-\mathrm{Sp} \log _{2} \mathrm{p}$, donde $\mathrm{p}=$ proporción de cada especie dentro del total. Se calculó el índice de Simpson ( $\left.D^{\prime}\right)$ que varía entre 0 y 1 , cuya fórmula es $D^{\prime}=1-\Sigma(\mathrm{ni} / N)^{2}$, donde ni: número de individuos en la zona evaluada, siendo el valor equivalente a 1 como el de máxima diversidad [18]. Las muestras de fitoplancton se obtuvieron al filtrar 20 litros de agua, utilizando una malla de $30 \mu \mathrm{m}$ de abertura, en cada estación de muestro, cuyo filtrado fue depositado en frascos de plástico de $500 \mathrm{ml}$. la muestra se conservó con formaldehido al 40\% y neutralizado con tetraborato de sodio hasta llegar un $\mathrm{pH} 7-7.3$ y almacenadas en un lugar fresco $\left(<15^{\circ} \mathrm{C}\right)$ protegidas de la luz [29] [39]. En el laboratorio Ambiental SGS del Perú se discriminaron e identificaron los organismos el nivel taxonómico y recuentos algales, según la metodología de Wehr \& Scheath y Bicudo \& Meneses [40]. 


\section{Resultados y discusión}

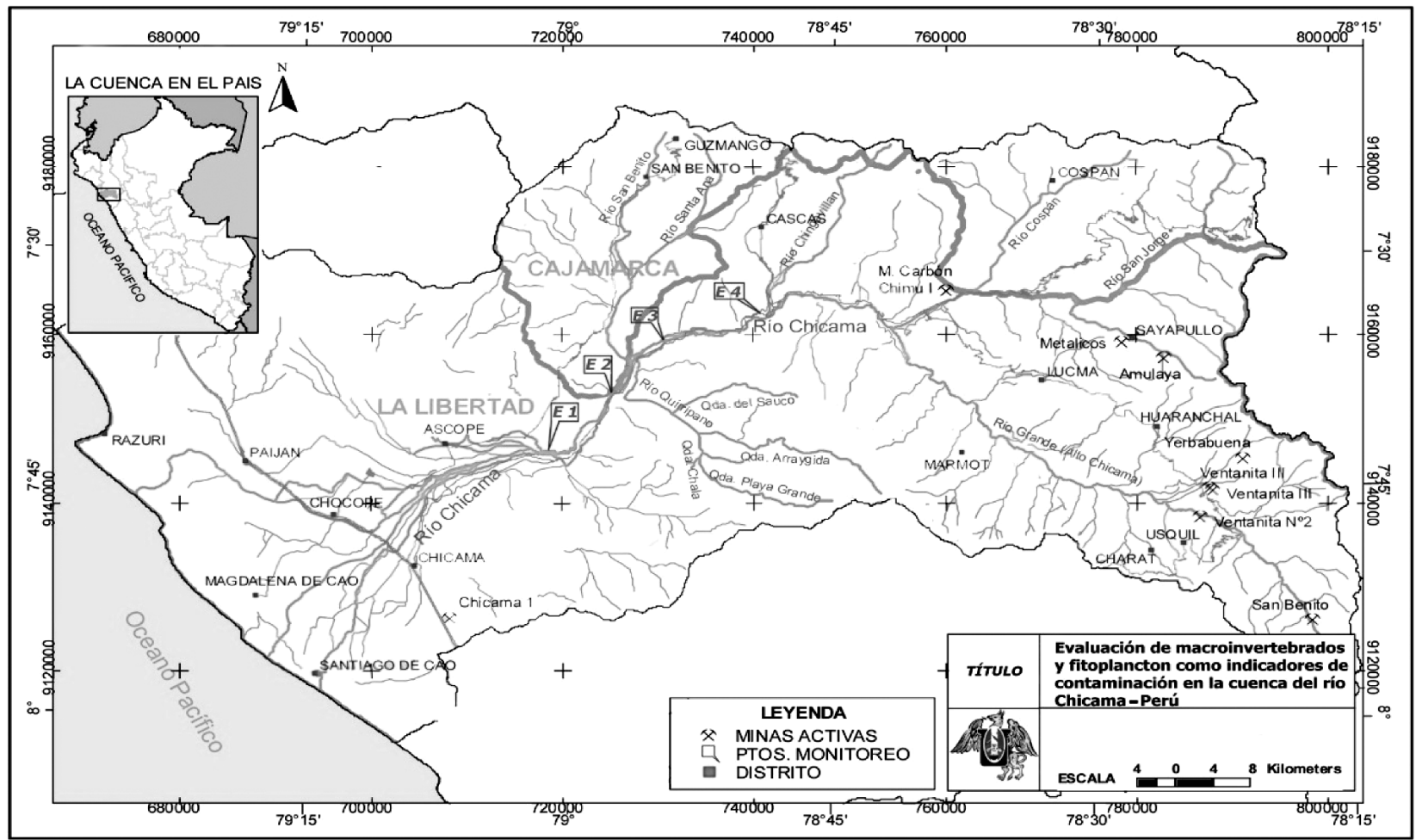

Figura 1. Ubicación de estaciones de muestreo en la cuenca del río Chicama, Perú.

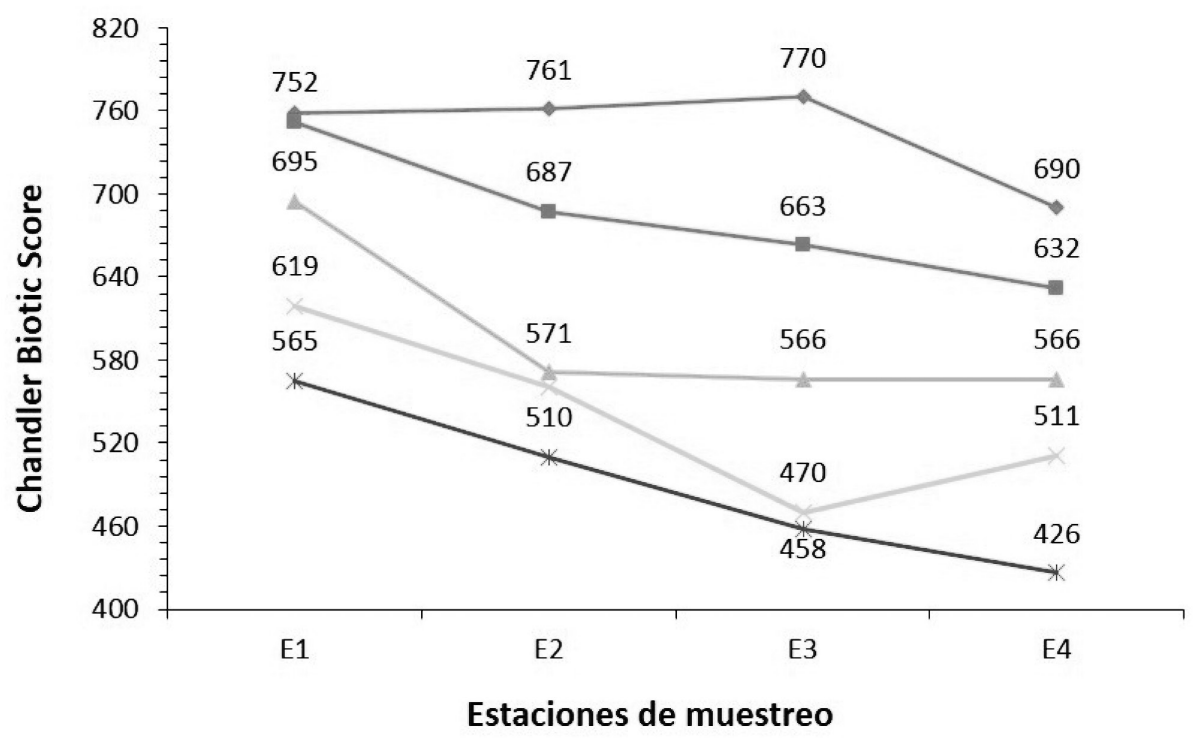

$\multimap 04 / 05 / 2015 \rightarrow-05 / 06 / 2015 \longrightarrow 05 / 07 / 2015 \longrightarrow 06 / 08 / 2015 \multimap 05 / 09 / 2015$

Figura 2. Determinación del índice biótico Chandler Biotic Score (CBS) en las estaciones de muestreo en la cuenca del río Chicama, Perú. 


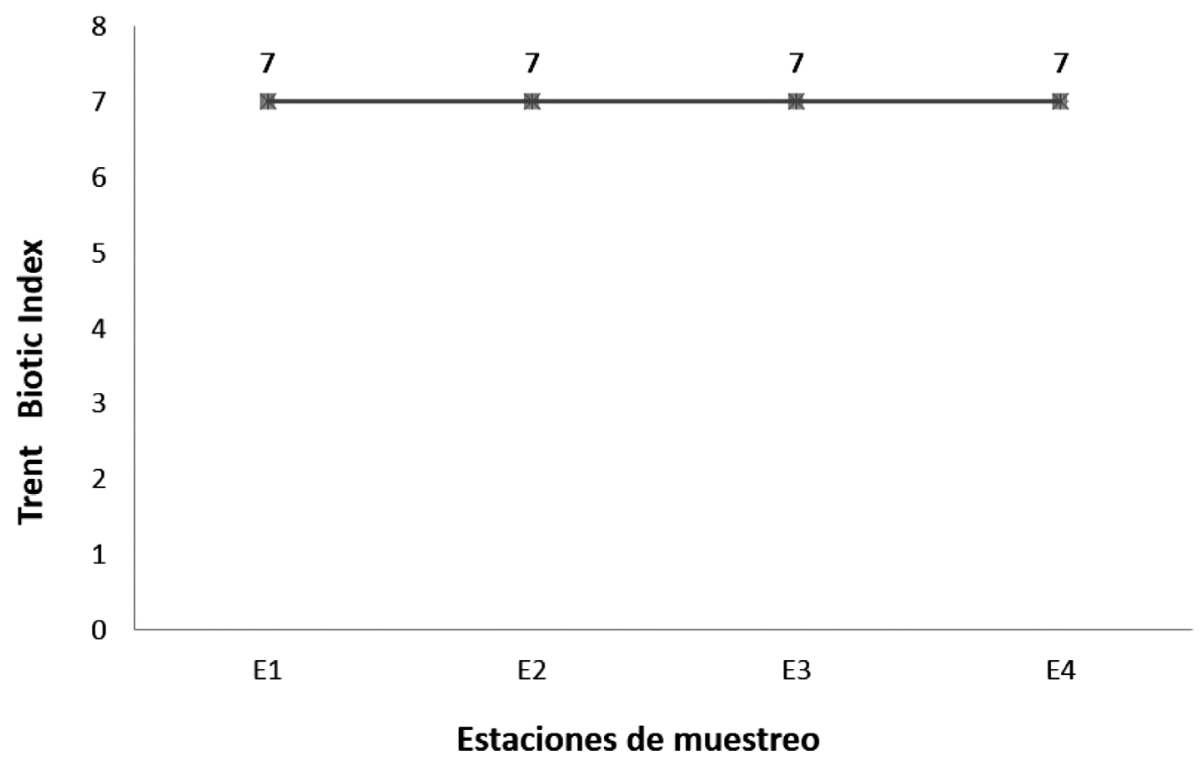

$\longrightarrow 04 / 05 / 2015 \rightarrow-05 / 06 / 2015 \longrightarrow 05 / 07 / 2015 \multimap 06 / 08 / 2015 \longrightarrow 05 / 09 / 2015$

Figura 3. Determinación del índice biótico Trent Biotic Index (TBI) en las estaciones de muestreo en la cuenca del río Chicama, Perú.

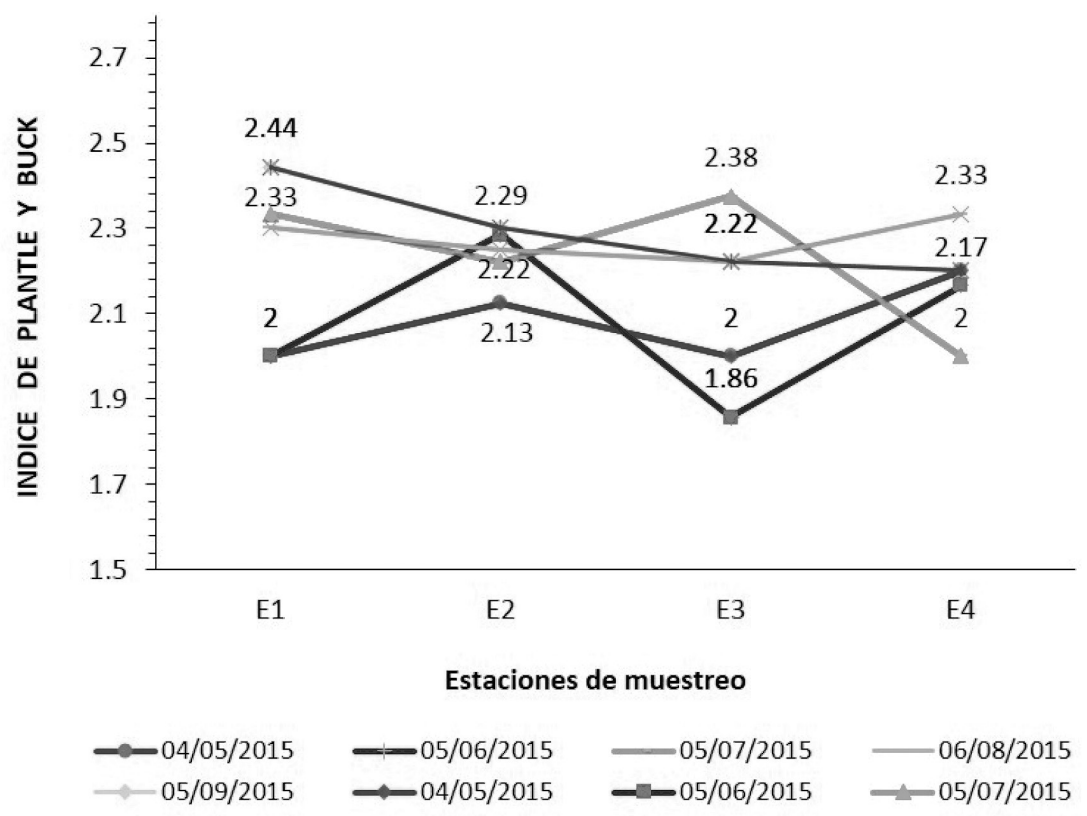

Figura 4. Determinación del índice saprobio Pantle y Buck en las estaciones de muestreo en la cuenca del río Chicama, Perú. 
Cuadro 1. Coeficiente de correlación de Spearman entre la abundancia los taxones y el índice de Pantle y Buck.

\begin{tabular}{|c|c|c|}
\hline \multicolumn{2}{|c|}{ INDICE DE PANTLE Y BUCK } \\
\hline TAXONES & Coeficiente de Correlación de Spearman & Valor de significancia \\
\hline Cocconeis placentula &,$- 615^{* *}$ &, 004 \\
\hline Nitzschia linearis &,- 393 &, 086 \\
\hline Nitzschia obtusa &,- 095 &, 690 \\
\hline Cymbella minuta &, 100 &, 675 \\
\hline Gomphonema sp. &, 406 &, 076 \\
\hline Rhoicosphenia sp. &,- 249 &, 289 \\
\hline Fragilaria capucina &,- 316 &, 174 \\
\hline Meridion sp. &, 308 &, 186 \\
\hline Synedra ulna &, $490^{*}$ &, 028 \\
\hline Tabellaria flocculosa &, $487^{*}$ &, 029 \\
\hline Scenedesmus acutus &, $569^{* *}$ &, 009 \\
\hline Zygnema pectinatum &, $507^{*}$ &, 023 \\
\hline Mougeotia sp. &, $693^{* *}$ &, 001 \\
\hline Cosmarium botrytis &, $608^{* *}$ &, 004 \\
\hline${ }^{* *}$ Correlation is significant at the 0.01 level $\left(2-\right.$ tailed). ${ }^{*}$ Correlation is significant at the 0.05 level (1-tailed). \\
\hline
\end{tabular}

Cuadro 2. Coeficiente de correlación de Spearman entre la abundancia los taxones y Chandler Biotic Score

\begin{tabular}{|c|c|c|}
\hline \multicolumn{3}{|c|}{ CHANDLER BIOTIC SCORE } \\
\hline TAXONES & Coeficiente de Correlación de Spearman & Valor de significancia \\
\hline Tricorythodes $s p$ &, $860^{* *}$ & 0,000 \\
\hline Leptohyphes $s p$ &, $590^{\star *}$ & 0,006 \\
\hline Thraulodes $s p$ &, $588^{* *}$ & 0,006 \\
\hline Moribaetis $s p$ &, $881^{* *}$ & 0,000 \\
\hline Baetis sp &, $649^{\star *}$ & 0,002 \\
\hline Clinotanypus $s p$ &,$- 485^{\star}$ & 0,030 \\
\hline Polypedilum $s p$ &, 096 & 0,686 \\
\hline Culex sp &,- 139 & 0,558 \\
\hline Simulium $s p$ &, 192 & 0,417 \\
\hline Atopsyche $s p$ &,- 258 & 0,273 \\
\hline Tropisternus $s p$ &, $636^{* *}$ & 0,003 \\
\hline Hidrophilidae $m s p$ &, 434 & 0,056 \\
\hline Centrocorisa $s p$ &,- 231 & 0,326 \\
\hline
\end{tabular}

Continua... 
...Continuacion

\begin{tabular}{|c|c|c|}
\hline \multicolumn{3}{|c|}{ CHANDLER BIOTIC SCORE } \\
\hline TAXONES & Coeficiente de Correlación de Spearman & Valor de significancia \\
\hline Nerthra sp &,- 139 & 0,558 \\
\hline Microvelia sp &,- 130 & 0,584 \\
\hline Rhagovelia sp & ,034 & 0,887 \\
\hline Veliidae msp &,- 355 & 0,125 \\
\hline Hydromera sp &,- 423 & 0,063 \\
\hline Limnogonus sp &,- 270 & 0,249 \\
\hline Limnocoris sp &, $717^{\star \star}$ & 0,000 \\
\hline Coridalus sp &, $720^{\star *}$ & 0,000 \\
\hline Sympetrum sp &,$- 775^{\star \star}$ & 0,000 \\
\hline Hidrachnidae msp &,- 231 & 0,328 \\
\hline Tubifex sp. &,- 139 & 0,558 \\
\hline
\end{tabular}

Cuadro 3: Coeficiente de correlación de Spearman entre los parámetros fisicoquímicos y el índice de Pantle y Buck

\begin{tabular}{|c|c|c|c|c|}
\hline \multirow[b]{2}{*}{$\begin{array}{l}\text { Parámetros físico } \\
\text { químicos }\end{array}$} & \multicolumn{2}{|c|}{ ÍNDICE DE PANTLE Y BUCK } & \multicolumn{2}{|c|}{ CHANDLER BIOTIC SCORE } \\
\hline & $\begin{array}{l}\text { Coeficiente de } \\
\text { Correlación de } \\
\text { Spearman }\end{array}$ & $\begin{array}{c}\text { Valor de } \\
\text { significancia }\end{array}$ & $\begin{array}{l}\text { Coeficiente de } \\
\text { Correlación de } \\
\text { Spearman }\end{array}$ & $\begin{array}{c}\text { Valor de } \\
\text { significancia }\end{array}$ \\
\hline Temperatura $\left({ }^{\circ} \mathrm{C}\right)$ &,- 098 & ,682 &,$- 479^{*}$ & ,033 \\
\hline Caudal (m³/seg.) &,$- 512^{*}$ & 021 & ,332 &, 152 \\
\hline $\begin{array}{l}\text { Conductividad } \\
\text { eléctrica. }(\mu \mathrm{S} / \mathrm{cm})\end{array}$ & ,691" &, 001 &,$- 569 * *$ & ,009 \\
\hline SDT (mg/L) & $695^{*}$ & ,001 &,$- 691^{\star \star}$ & ,001 \\
\hline $\mathrm{pH}$ &, $547^{*}$ & 013 &,$- 841^{\star \star}$ & ,000 \\
\hline $\mathrm{PO} 4-3$ (mg/L) &, $522^{*}$ & ,018 &,$- 842^{\star *}$ & ,000 \\
\hline NO2- (mg/L) &, $533^{*}$ & ,016 &,$- 572^{\star \star}$ & ,008 \\
\hline NO3- (mg/L) & , 108 & ,649 & ,038 & 875 \\
\hline $\mathrm{DBO}^{5}(\mathrm{mg} / \mathrm{L})$ & 296 & ,205 &, 101 & 672 \\
\hline $\mathrm{OD}(\mathrm{mg} / \mathrm{L})$ &,$- 468^{*}$ & ,038 &, 197 &, 405 \\
\hline $\mathrm{sO} 4-3$ (mg/L) &,- 173 & ,466 &,- 087 & ,716 \\
\hline
\end{tabular}

La composición de la fauna bentónica encontrada en la cuenca del río Chicama estuvo constituida por 24 géneros, 18 familias y 9 ordenes, con predominancia de Baetis sp., Rhagovelia $s p$. y Atopsyche sp., en menor grado, en comparación con los anteriores, Tricorythodes sp., Clinotanypus sp., y Simulium sp. (cuadro 2). La riqueza y abundancia de especies como 
indicaron los resultados reportados fueron relativamente bajos, en comparación con el número de familias determinados en otros estudios realizados en la cuenca alta del río Chicama [24] [22] [41]. Dentro de los macroinvertebrados identificados, el orden Ephemeroptera y Trichoptera, por lo general se presentaron especies que viven en aguas corrientes, limpias y bien oxigenadas; donde algunas especies parecen resistir cierto grado de contaminación [42]. Del orden Ephemeroptera, Baetis sp. y Tricorythodes sp. (cuadro 2), se asocian a ecosistemas donde la contaminación es incipiente o nula [43] [3], permitiendo su asociación o correlación positiva con la concentración de fosfatos, nitritos y nitratos (cuadro 3). El orden Trichoptera es uno de los órdenes más importantes en las cadenas alimentarias de arroyos, por lo que son potenciales indicadores de contaminación, teniendo en cuenta que la mayor abundancia de esta taxa se presenta en aguas oligotróficas (en buen estado biológico), tal como refiere al precisar que el orden Trichoptera fue el más abundante en la cuenca de la quebrada Piedras Blancas, Antioquia - Colombia [44] [45] y en las cuencas alta y baja del río Gariché, provincia de Chiriquí, Panamá respectivamente, caracterizando a ambos sistemas lóticos como oligotróficos.

La distribución de órdenes de reconocida sensibilidad como Plecoptera, Ephemeroptera y Trichoptera se encontraron en la cuenca alta del río Chillán en Chile, mientras en la cuenca media y baja estas taxas presentaron baja abundancia [46]. En contraste, Atopsyche sp.(cuadro 2) es una de las especies que puede permanecer en aguas oligotróficas o con algunas trazas de contaminación [33], correlacionándose positivamente y significativamente $(p<0.05)$ con la cantidad de nitratos presente en el medio (cuadro 3). En contraste a lo anterior, solo algunas familias pertenecientes al orden Díptera, fisiológicamente pueden resistir altos grados de contaminación acuática, estos organismos son considerados como buenos indicadores de aguas de baja calidad [47]; donde la abundancia de Simuliidae, Culicidae (cuadro 2) y en especial Chironomidae es asociado frecuentemente en aguas contaminadas por materia orgánica [48], corroborando de esta manera los resultados presentados se determinó que Clinotanypus sp. y Polypedilum sp. se correlacionaron positivamente con la cantidad de nitritos $(p<0.05)$ y nitratos $(p<0.01)$, y negativamente con la cantidad de oxígeno disuelto $(p<0.05)$ (cuadro 3$)$; en tal sentido, su presencia en las cuatro estaciones de muestreo reflejaría una menor calidad del agua, debido a que los Chironomidae, aumentan en número en aquellos lugares con escasa concentración de oxígeno disuelto y mayor cantidad de partículas en suspensión, condiciones características de zonas que han sido afectados o estresados por el corte total del bosque de la ribera [49], y que se observó claramente en las estaciones de muestreo.

La calidad de agua de la cuenca del río Chicama evaluado reflejó un nivel "ligeramente aceptable" correspondiente a ríos con aguas levemente contaminadas [50], representados mediante el Chandler Biotic Score (figura 2) con una mínima puntuación de 426 en la estación (E 4) y el Trent Biotic Index (figura 3) con un valor de 7, los cuales fueron comparables con el valor (62) determinado mediante el Índice Biótico Andino (ABI) [24] para el sector Puente Ochape, correspondiente a la estación de muestreo (E 4) para el presente estudio, lo que permitió caracterizar como aguas de calidad regular. En contraste, el índice saprobio de Pantle y Buck (figura 4) evaluado indicó que la cuenca se encontró dentro del rango de $\alpha$-mesosaprobio (aguas moderadamente contaminadas), con una puntuación mínima de 1.6 en la estación 3 y una máxima de 2.44 en la estación de muestreo (E 1). En relación a lo anterior y según el análisis de correlación de Spearman, la abundancia de Synedra ulna, Tabellaria flocculosa, Scenedesmus acutus, Zygnema pectinatum, Mougeotia sp, y Cosmarium botrytis (cuadro 1) presentaron correlación positiva y estadísticamente significativa $(p<0.5)$ con el Indice de Pantle y Buck; en contraste, Cocconeis placentula tuvo una correlación negativa y estadísticamente significativa $(p<0.01)$ con este índice. Entre parámetros fisicoquímicos evaluados la cantidad de conductividad eléctrica, sólidos totales disueltos, $\mathrm{pH}$, fosfatos y nitritos, guardaron correlación positiva con el índice saprobio de Pantle y Buck (cuadro 3); sin embargo, la cantidad de oxígeno 
disuelto y de caudal guarda una correlación negativa y estadísticamente significativa $(p<0.05)$ con este índice. Donde el aumento del caudal en el presente estudio, se consideraría como un factor limitante en el desarrollo de la flora planctónica, en especial de aquellas indicadoras de contaminación, limitando la disponibilidad y aprovechamiento de los nutrientes.

En lo referente a la flora planctónica encontrada en la cuenca del río Chicama, estuvo constituida principalmente por diatomeas (Bacillariophyta), y en menor proporción por especies pertenecientes a la clase Chlorophyta, resultados concordantes al determinar que las diatomeas (Bacillariophytas) obtuvo la mayor frecuencia en todas las estaciones de muestreo analizados en los ríos El Perejil, Caballo Moro y Chuyuhual; tributarios en la cuenca alta del río Chicama [51]; del mismo modo estudios realizados en los ríos Cuarto y Piedra Blanca (Córdoba, Argentina) respectivamente [52] [53], cuyos rangos altitudinales de las zonas de estudio fueron muy similares. Las especies que predominaron inicialmente en la mayoría de las estaciones de muestreo fueron: Nitzschia linearis, Fragilaria capucina y Gomphonema sp. (cuadro 1), las cuales en el transcurso de tiempo de la evaluación presentaron una abundancia de especies disminuida. La diversidad del fitoplancton de acuerdo a los índices utilizados fue en valores promedio; en consecuencia, la riqueza y abundancia de especies como lo demuestran los valores reportados fueron relativamente bajos [54], cuyos rangos altitudinales de las zonas de estudio fueron muy similares.

Una comunidad bajo la presión de la contaminación se caracteriza por poseer un bajo número de especies con un gran número de individuos; que en contraste, una comunidad natural se caracteriza por presentar una gran diversidad de especies y un bajo número de individuo por especies [48] [55]. En base en lo anterior y según los resultados de la diversidad del fitoplancton presente en el río Chicama indicó que existe contaminación importante; aún que la diversidad resulta muy atractiva para determinar la calidad de agua, sus resultados pueden variar con el método de muestreo, la naturaleza del sustrato [56] y la época del año [48]. El análisis de correlación de Spearman indicó que la abundancia de Tricorythodes sp., Leptohyphes sp., Thraulodes sp., Moribaetis sp., Baetis sp., Limnocoris sp., Coridalus sp., Sympetrum sp. y Clinotanypus sp.(cuadro 2) se encontró correlación estadísticamente significativa $(r=0,860, r=0,590, r=0,588, r=0,881, r=0,649, r=-0,485, r=0.636, r=0.717, r=0.720$ y $r=-0.775$ respectivamente) con Chandler Biotic Score $(p<0.05)$, mientras que la abundancia del resto de los taxones son independientes de este índice. Asimismo, la conductividad eléctrica, sólidos totales disueltos, $\mathrm{pH}$, fosfatos, nitritos y temperatura, esta última considerando un nivel de significancia de $5 \%$, presentó una correlación negativa y estadísticamente significativa $(p<0.01)$ con Chandler Biotic Score (cuadro 3), entendiendo que a medida que los valores de estos parámetros fisicoquímicos se incrementen, permitirán la abundancia de taxas de dípteros, en especial Chironomidae, y la ausencia de taxas de Ephermeropteros y Trichopteros, resultando en la diminución del valor de Chandler Biotic Score.

El fitoplancton, a diferencia de los macroinvertebrados, su distribución está sometida a una sucesión estacional, el origen del sistema lótico, el tamaño, forma y superficie del espejo de agua, profundidad, naturaleza del fondo, radiación solar, estado trófico, entre otras variables fisicoquímicas; los cuales son factores que influyen sobre el crecimiento y desarrollo de distintas especies algales y sus variaciones temporales; en semejanza con los macroinvertebrados esta sucesión es dependiente de las perturbaciones presentes en el medio, generalmente de origen antrópico, que pueden dar lugar a un proceso de eutrofización como resultado de una mayor incorporación de nutrientes y materia orgánica disuelta o particulada [52] [53]. En tal sentido, se encontró que la presencia y abundancia de especies pertenecientes a la división Cyanophyta (cuadro 1) se relacionó significativamente con cantidades elevadas de nitratos (cuadro 3), no presentando asociación significativa con las Chlorophyta o Bacillariophyta; en contraste con los resultados, se determinó que Scenedesmus acutus, Zygnema pectinatum y Cosmarium botrytis 
(cuadro 1), pertenecientes a la división Chlorophyta, presentaron una correlación positiva con la concentración de nitratos, fosfatos y sulfatos presentes en el agua (cuadro 3), entendiendo que a mayor aumento de la concentración de estos nutrientes mayor será la abundancia de dichas especies [57] [58].

Estudios realizados en el río Duwamish, Estados Unidos se encontró que la abundancia del fitoplancton fue fuertemente afectado por la presencia de nitratos, que por los fosfatos, indicando que el nitrato era el factor limitante para el crecimiento del fitoplancton en ese sistema [57]; sin embargo, en investigaciones sobre distribución temporal del fitoplancton [52], en un lago urbano del centro de Argentina (río Cuarto, Córdoba) se determinó que la temperatura, pH y conductividad fueron los factores fisicoquímicos que determinaron la distribución y abundancia temporo-espacial de las especies. En contraste, en la presente investigación se determinó que sólo la abundancia de Tabellaria flocculosa, Scenedesmus acutus, Zygnema pectinatum y Cosmarium botrytis (cuadro 1), se relacionó con la concentración de fosfatos presentes en el agua (cuadro 3); sin embargo, la abundancia de las especies determinadas se relacionaron negativamente y estadísticamente significativa $(p<0.01)$ con la cantidad de sulfatos, como fue el caso de Synedra ulna; se han realizados estudios realizados en Synedra ulna, donde se ha demostrado que es un buen indicador en la determinación de grados de eutrofización, de leve a moderado [59] [60]. Por otro lado, se concluyó que los compuestos carbonados y sulfurados no serían nutrientes limitantes.

\section{Conclusiones}

- En cuanto a la calidad de agua; medido con los índices biológicos, Chandler Biotic Score, Trent Biotic Index y el Índice de Pantle y Buck, y los parámetros físicosquímicos evaluados, se determinó que la sección evaluada de la cuenca baja del río Chicama indica que existe un leve deterioro de la calidad de sus aguas.

- Los macroinvertebrados en la cuenca del río Chicama estuvo constituida por 24 géneros, 18 familias y 9 ordenes, con predominancia de Baetis sp, Rhagovelia sp y Atopsyche $s p$. El fitoplancton en la cuenca del río Chicama estuvo constituida por 14 especies, 9 familias y 7 ordenes, con relativa predominancia de Gomphonema sp. y Scenedesmus acutus.

- En cuanto a la calidad de agua; medido con los índices biológicos, Chandler Biotic Score Trent Biotic Index y el índice de Pantle y Buck. Los macroinvertebrados y fitoplancton resultaron organismos ideales para la evaluación y monitoreo de los cuerpos de agua, con predominancia de contaminación de tipo orgánico.

\section{Referencias}

[1] R. Kolkwitz and M. Marsson. Okoligie der pflanzlichen Saprobien. Berichte der Deutschen Botanischen Gesellschaft, 1908, 26A:505-519.

[2] R. Kolkwitz and M. Marsson. Okologie der tierischen Saprobien. Beitrage zur Lehre von des biologischen Gewasserbeurteinlung. Internationale Revue der Gesamten Hydrobiologie und Hydrographie, 1909, 2:126152.

[3] R. Barba-Álvarez, G. De La Lanza-Espino, A. Contreras-Ramos, and I. González-Mora. Insectos acuáticos indicadores de calidad del agua en México: casos de estudio, ríos Copalita, Zimatán y Coyula, Oaxaca. Revista Mexicana de Biodiversidad, 2013, 84:381-383.

[4] R. Figueroa, C. Valdovinos, E. Araya, and O. Parra. Macroinvertebrados bentónicos como indicadores de calidad de agua de ríos del sur de Chile. Revista Chilena de Historia Natural, 2003, 76:275-285. 
[5] N. Prat, A. Munné, N. Bonada, C. Solá, M. Llanos, M. Rieradevall, R. Casanovas, and M. Vila. La calidad ecológica del Llobregat, Besòs, el Foix y Tordera. Informe 1999. Estudios de la calidad ecológica de los ríos, 2001, pp. 9-171.

[6] H. Hynes. The biology of polluted waters.Liverpool Univ. Press, 1960, pp. 202.

[7] H. Hawkes. Origin and development of the biological monitoring working party score system. Wat. Res., 1997, 32(3):964-968.

[8] V. Resh, R. Norris, and M. Barbour. Design and implementation of rapid assessment approaches or water resource monitoring using benthic macroinvertebrates. Aust. J. Ecol., 1995, 20:108-121.

[9] J. Alba. Macroinvertebrados acuáticos y calidad de las aguas de los ríos. IV Simposio del Agua en Andalucía (SIAGA). Almería, España, 1996, 2:203-213.

[10] C. Townsend, M. Scarsbrook, and S. Dolédec. Quantifying disturbance in streams: alternative measures of disturbance in relation to macroinvertebrate species traits and species richness. Journal of the North American Benthological Society, 1997, 16:531-544.

[11] J. Plafkin, L. Barbour, K. Porter, and R. Hughes. Rapid bioassessment protocols for use in streams and rivers: benthic macroinvertebrates and fish. U.S. Environmental Protection Agency. EPA 440/4-89/001, 8 chapters, Appendices A-D, 1989.

[12] M. Barbour. The re-invention of biological assessment in the U.S. Human and Ecological Risk Assessment, 1997, 3:933-940.

[13] J. Wrigth, D. Moss, P. Armitage, and M. Furse. A preliminary classification of running water sites in Great Britain based on macroinvertebrate species and the prediction of community type using environmental data. Freshwater Biology, 1984, 14:221-256.

[14] J. Wrigth, D. Sutclife, and M. Furse. Assessing the biological quality of fresh waters: RIVPACS and other techniques. Freshwater Biological Association, Cumbria, United Kingdowm, 2000, pp. 23.

[15] N. Bonada. Ecology of the macroinvertebrate communities in Mediterranean rivers at different scales and organization levels. Tesis de grado para optar el grado de Doctor en Ciencias. Departamento de Ecología, Facultad de Ciencias, Universidad de Barcelona, 2003.

[16] D. Rosenberg, and V. Resh. Introduction to freshwater biomonitoring and benthic macroinvertebrates. In Freshwater biomonitoring and benthic macroinvetebrates, D. M. Rosenberg y V. H. Resh (eds). Chapman and Hall, New York, 1993, pp. 1-9.

[17] E. Peña, M. Palacios, and N. Ospina. Algas como indicadoras de contaminación. Editorial Universidad Del Valle $1^{\text {era }}$ edición. Cali- Colombia, 2005, pp. 51-104.

[18] M. Lara, J. Moreno, and E. Amaro. Fitoplancton: conceptos básicos y técnicas de laboratorio. México: Universidad Autónoma Metropolitana Iztapalapa, 1996, pp. 227.

[19] A. M. Guerrero. Macroinvertebrados como bioindicadores en la evaluación de la calidad de agua en ríos. Rev. Tecnología en Marcha, 1993, 12(3): 39-54.

[20] C. Paredes, J. Lannacone, and L. Alvariño. Uso de macroinvertebrados bentónicos como indicadores de calidad de agua del río Rímac, Lima - Callao, Perú. Revista Colombiana de Entomología, 2005, 31(2):219-225.

[21] A. Gómez, C. Medina, A. Ramírez, L. Pollack, and H. Castillo. Macroinvertebrados bentónicos y parámetros fisicoquímicos como indicadores de calidad de agua de la cuenca media del río Moche. La Libertad.2010. Revista Rebiol, 2010, 30(2):79-84.

[22] C. Medina. Estado ecológico del río Chicama. Regiones. La Libertad y Cajamarca Perú 2006. Tesis para adoptar el grado de doctor en medio ambiente. Escuela de postgrado. Universidad Nacional de Trujillo, 2007, pp. 1-91.

[23] C. Medina, J. Balmaceda, R. Ramírez, F. Peláez, W. Reyes, and J. Puche. Caracterización físico-química y microbiológica del río Chicama. Regiones la Libertad y Cajamarca, Perú. 2006. Revista SCIENDO, 2007, 10(2):31-40.

[24] J. Balmaceda and J. Puche. Macroinvertebrados bentónicos como bioindicadores de calidad de agua en río Región La Libertad y Cajamarca. Perú. Revista SCIENDO, 2007, 10(2):41-52.

[25] E. Ortega. El fitoplancton como indicador de la calidad de agua del lago Huayamilpas, Del. Coyoacán, D.F. Tesis para adoptar el grado de Lincenciado en Hidrología. Universidad Autónoma Metropolitana Iztapalapa. Mexico, 1996, pp. 2-15.

[26] A. Luján. Las algas indicadoras de la calidad de agua. Interciencia, revista de ciencias naturales. Universidad Nacional Rio Cuarto, 2000, 4(4):4-8. 
[27] C. Seeligmann, B. Tracanna, S. Martínez, and S. Isasmendi. Algas fitoplanctónica en la evaluación de la calidad de agua de sistemas loticos en el noroeste Argentino. Rev. Limnetica, 2001, 20(1):123-133.

[28] C. Prosperi, C. Rodríguez, M. Pierotto, M. Mancini, C. Daga, and M. Gonella. Evaluación de la contaminación y eutrofización de aguas superficiales de la provincia de Córdoba. Temas de Ciencia y Tecnología, 2005, 2(7):4.

[29] American Public Health Association (APHA). Standard methods for examination of water and wastewater. APHA-AWWA-WPCF, Washington, USA, 2012, pp. 1496.

[30] Hynes H. The Ecology of Running Waters.Liverpool University Press, England, 1970, pp. 555.

[31] W. Hilsenhoff. Rapid field assessment of organic pollution with a family level biotic índex. J. North Amer. Benthol. Soc., 1988, 7:65-68.

[32] D. Lenat and M. Barbour. Using benthic macroinvertebrate community structure or rapid, cost-effective, water quality monitoring: rapid bioassessment En: Loeb SL \& A Spacie (eds) Biological monitoring of aquatic systems, 1994, pp. 187-215.

[33] E. Domínguez, M. Hubbard, and W. Peters. Claves para ninfas y adultos de las familias y géneros de Ephemeroptera (Insecta) Sudamericanos. Instituto de Limnología "Dr, Raúl A. Ringuelet", Argentina. Biología acuática, 1992, pp. 16-36.

[34] H. Fernández and E. Domínguez. Guía para la determinación de artrópodos bentónicos sudamericanos. Editorial Universitaria de Tucumán, Tucumán, Argentina, 2002, pp. 282.

[35] W. Fischer, F. Krupp, W. Schneider, C. Sommer, K. Carpenter, and V. Niem. Guía para la identificación de especies para los fines de la pesca. Pacífico Centro- Oriental. Volumen I: Plantas e Invertebrados. FAO. Roma, 1995, pp. $12-68$.

[36] J. Needham and P. Needham. Los seres vivos de las aguas dulces. Traducción adaptada para España y América. Editorial Reverté. Barcelona, España, 1978.

[37] E. Ayana. Estudio de la calidad del agua del río Babahoyo y sus afluentes: Índice Saprobio. Tesis para optar el grado de Magister en Ciencias: Manejo Sustentable de Recursos Bioacuáticos y Medio Ambiente. Facultad de Ciencias Naturales. Universidad de Guayaquil, 2013, pp.1-70.

[38] J. Zar. Biostatistical analysis. Upper Saddle River, Prentice-Hall. $3^{\text {th }}$ Ed., 1996, pp. 662.

[39] Confederación Hidrográfica de Ebro. Metodología para el establecimiento del estado ecológico según la directiva marco del agua. Protocolos de muestreo y análisis de fitoplancton, 2005, pp. 17-28.

[40] A. Sardi-Saavedra, E. Peña-Salamanca, C. Madera-Parra, and V. Cerón-Hernández. Diversidad de las comunidades de algas asociadas a un sistema algal de alta tasa fotosintética para la biorremediación de lixiviados de rellenos sanitarios. Lat. Am. J. Aquat. Res., 2016, 44(1):113-120.

[41] C. Medina, M. Hora, I. Asencio, W. Pereda, and R. Gabriel. El Índice Biological Monitoring Working Party (BMWP), modificado y adaptado a tres microcuencas del Alto Chicama. La Libertad. Perú. 2008. Rev. Sciendo, 2010, 13(2):1-15.

[42] D. Vergara, Entomofauna lótica bioindicadora de la calidad del agua. Tesis de grado para optar al título de Magíster de Ciencias en Medio Ambiente y Desarrollo, 2009, pp. 1-77.

[43] P. Bispo, C. Froehlich, and L. Oliveira. Stonefly (Plecóptera) fauna of stream in a mountainous area of central Brazil: abiotic factors and nymph density, Revista Brasileira de Zoologia, 2002, 19(1):325-334.

[44] G. Posada, P. Roldan, and J. Ramírez. Caracterización físico-química y biológica de la calidad de aguas de la cuenca de la quebrada Pi edras Blancas, Antioquia, Colombia. Revista de Biología Tropical, 2000, 48(1):5970.

[45] J. Guinard, T. Ríos, and J. Bernal. Diversidad y abundancia de macroinvertebrados acuáticos y calidad del agua de las cuencas alta y baja del río Gariché, provincia de Chiriquí, Panamá. Rev. Gestión y Ambiente, 2013, 16(2):61-70.

[46] R. Figueroa, A. Palma, V. Ruíz, and X. Niell. Análisis comparativo de índices bióticos utilizados en la evaluación de la calidad de las aguas en un río mediterráneo de Chile: río Chillán, VII Región. Revista Chilena de Historia Natural, 2007, 80:225-242.

[47] Gamboa M., R. Reyes \& J. Arrivillaga. Macroinvertebrados bentónicos como bioindicadores de salud ambiental. Boletín de Malariología y salud ambiental, 2008, 48(2):109-120.

[48] G. Roldán. Los macroinvertebrados y su valor como indicadores de calidad de agua. Rev. Acad. Columb. Cienc., 1999, 23(88):375-387. 
[49] M. Callisto, F. Barbosa, and P. Moreno. Macrobenthic diversity on different habitats in an altitudinal lake surrounded by Eucalyptus plantations and secondary Atlantic Forest (southeast Brazil). Acta Limnologica Brasiliensia, 2000, 12(1): 55-61.

[50] D. Muñoz, J. Giacometti, and J. Ortiz. Caracterización de macroinvertebrados acuáticos de la cuenca alta del río Pita, Cantón Rumiñahui-Ecuador, 2011, [Online]. Available: http://www.espe.edu.ec/portal/ files/sitio_congreso_2011/papers/V8.pdf

[51] K. Sánchez. Microalgas de los ríos: El Perejil, El Caballo Moro y El Chuyuhual, La Libertad, Perú. 2012. Tesis par optar el Título de Biólogo. Escuela de Ciencias Biológicas. Facultad de Ciencias Biológicas. Universidad Nacional de Trujillo, 2012, pp.1-47.

[52] M. Novoa, A. Martínez, M. Luque, and D. Lombardo. Distribución temporal del fitoplancton en un lago urbano del centro de argentina (Río Cuarto, Cordoba). Rev. Biológicas, 2011, 13(2):1-14.

[53] M. Luque, and A. Martínez. Distribución temporal del fitoplancton y epifiton en el río Piedra Blanca (Córdoba, Argentina). Limnética, 2003, 22 (3-4):19-34

[54] P. Castañe, C. Loez, H. Olguín, A. Puig, M. Rovedatti, M. Topalián, and A. Salibián. Caracterización espacial de parámetros fisicoquímicos y del plancton en un río contaminado (río Reconquista, Argentina). Rev. Int. Contaminación Ambiental, 1998, 14(2):69-77.

[55] N. Bonada, N. Prat, V. Resh, and B. Statzner. Developments in aquatic insect biomonitoring: a comparative analysis of recent approaches. Annual Review of Entomology, 2006, 51:495-523.

[56] E. Morelli and A. Verdi. Diversidad de macroinvertebrados acuáticos en cursos de agua dulce con vegetación ribereña nativa de Uruguay. Revista Mexicana de Biodiversidad, 2014, 85:1160-1170.

[57] H. Ozbay. Composition and abundance of phytoplankton in relation to physical and chemical variables in The Kars River, Turkey. Rev. Phyton, 2011, 80(1):85-92.

[58] J. Ponce and I. Arredondo. Aporte al conocimiento limnológico de un embalse temporal tropical por medio de la aplicación de modelos multivariados. Anales del Instituto de Ciencias del Mar y Limnología. Universidad Nacional Autónoma de México, 1986, 13(2):46-66.

[59] M. Sosa, M. Novoa, and A. De Fabricius. Ficoflora de la cuenca endorreica fluviolacustre Chucul (Córdoba, Argentina). Rev. Biológicas, 2011, 13(1):14-23.

[60] P. Gadea, Variación espacial del fitoplancton en la zona costera de Gandía y su relación con la entrada de nutrientes. Tesis doctoral, Universidad Politécnica de Valencia, Departamento de Ingeniería Hidráulica y Medio Ambiente, 2009, pp. 11. 